\title{
EGOROFF PROPERTIES AND THE ORDER TOPOLOGY IN RIESZ SPACES $\left({ }^{1}\right)$
}

BY

\author{
THERESA K. Y. CHOW DODDS
}

ABSTRACT. In this paper we prove that, for a Riesz space $L$, the order closure of each subset of $L$ coincides with its pseudo order closure if and only if the order closure of each convex subset of $L$ coincides with its pseudo order closure; moreover, each of these statements is equivalent to the strong Egoroff property. For Archimedean Riesz spaces, similar results hold for the relative uniform topology.

1. Introduction. The Egoroff property arises naturally in the study of Riesz spaces and Boolean algebras, and it has been investigated by J. A. R. Holbrook in [4] where the relation between the Egoroff property and the classical Egoroff theorem (see [3]) is discussed in detail. In this paper we discuss the relation between the Egoroff property and the order topology in both settings. In $\$ 2$, we show that in a Boolean algebra $B$ the Egoroff property and the diagonal property for order convergence are each equivalent to the statement that the order closure of each subset of $B$ coincides with its pseudo order closure (sequential order closure). For Riesz spaces, a variant of the Egoroff property is introduced which we call the strong Egoroff property. We show in $\$ 3$ that the strong Egoroff property in Riesz spaces is, in fact, equivalent to the diagonal property for order convergence which has been discussed by many authors and goes back to L. Kantorovitch (see Satz 24 of [5]); furthermore, a Riesz space $L$ has the strong Egoroff property if and only if the order closure of each convex subset of $L$ coincides with its pseudo order closure. For Archimedean Riesz spaces, similar results relating to the relative uniform topology are obtained in $\$ 4$. Here the role of the strong Egoroff property is played by a strictly weaker property, which we call the $\sigma$-property.

2. The Egoroff property and order convergence in Boolean algebras. In this section $B$ will always denote a nondegenerate Boolean algebra. The elements

Received by the editors March 12, 1971 and, in revised form, April 13, 1973.

AMS (MOS) subject classifications (1970). Primary 46 A40.

Key words and phrases. Riesz spaces, Egoroff property, order convergence, pseudo order closure, relative uniform convergence.

(1) The results in this paper form part of the author's $\mathrm{Ph}$. $\mathrm{D}$. thesis submitted to the California Institute of Technology under the supervision of Professor W. A. J. Luxemburg. Copyright 1 1974, American Mathematical Society 
of $B$ will be denoted by $a, b, \ldots$; the unit element by 1 ; the zero element by 0 . For arbitrary elements $a, b \in B, a \vee b$ and $a \wedge b$ are respectively the join and the meet of $a$ and $b$. For each $a \in B$, the complement of $a$ is denoted by $a^{\prime}$. A sequence $\left\{a_{n}: n=1,2, \ldots\right\}$ in $B$ is called increasing if $a_{1} \leq a_{2} \leq \ldots$ and will be denoted by $a_{n} \uparrow_{n}$ or $a_{n} \uparrow$; the sequence is called decreasing if $a_{1} \geq a_{2} \geq \cdots$ and will be denoted by $a_{n} \downarrow_{n}$ or $a_{n} \downarrow$. If $a_{n} \uparrow$ and $a \in B$ is such that $a=\bigvee_{n} a_{n}$, then we write $a_{n} \uparrow a ; a_{n} \downarrow a$ is defined similarly. We write $a_{n k} \uparrow_{k} a_{n} \uparrow a$ if the sequence $\left\{a_{n}: n=1,2, \ldots\right\}$ satisfies $a_{n} \uparrow a$ and, for each $n$, the sequence $\left\{a_{n k}: k=1,2, \ldots\right\}$ satisfies $a_{n k} \uparrow_{k} a_{n} ; a_{n k} \downarrow_{k} a_{n} \downarrow a$ is defined similarly.

Definition 2.1. An element $a$ of a Boolean algebra $B$ is said to have the Egoroff property if, given any double sequence $\left\{a_{n k}: n, k=1,2, \ldots\right\}$, with $a_{n k} \uparrow_{k} a$ for each $n$, there exists a sequence $\left\{b_{m}: m=1,2, \ldots\right\}$ in $B$ such that $b_{m} \uparrow a$ and for every pair of indices $m, n$ there is an index $k(m, n)$ such that $b_{m} \leq a_{n, k(m, n)}$. A Boolean algebra is said to have the Egoroff property if every one of its elements has the Egoroff property.

It follows that a Boolean algebra $B$ has the Egoroff property if and only if its unit element 1 has the Egoroff property, since it is easy to show that, if an element $a$ in $B$ has that property and $c \leq a$, then $c$ has it also (if $c_{n k} \uparrow_{k} c$, consider $\left.a_{n k}=c_{n k} \vee\left(a \wedge c^{\prime}\right)\right)$.

The Egoroff property of Boolean algebras was first introduced by W. A. J. Luxemburg (see [9]).

Lemma 2.2. If $B$ is a Boolean algebra, then the following two statements are equivalent.

(1) $B$ bas the Egoroff property.

(2) If $a_{n k} \downarrow a_{n} \downarrow 0$ in $B$, then there exist $b_{m} \downarrow 0$ sucb that for every $m, b_{m} \geq$ $a_{n(m), k(m)}$ for some indices $n(m)$ and $k(m)$.

Proof. $(1) \Rightarrow(2)$ By complementation, the Egoroff property of 1 ensures that, if $c_{n k} \downarrow_{k} 0$, then there exists a sequence $c_{m} \downarrow 0$ such that $c_{m} \geq c_{n, k(m, n)}$ for all $m, n$. Let $c_{n k}=a_{n k} \wedge a_{n}^{\prime} ; c_{n k} \downarrow_{k} a_{n} \wedge a_{n}^{\prime}=0$ so that there are $c_{m}$ as above. Now $b_{m}=a_{m} \vee c_{m} \downarrow 0$ and $b_{m} \geq a_{m} \vee c_{m k}$, where $k=k(m, m)$. Thus $b_{m} \geq a_{m} \vee\left(a_{m k} \wedge\right.$ $\left.a_{m}^{\prime}\right)=\left(a_{m} \vee a_{m k}\right) \wedge 1=a_{m k}$.

(2) $\Rightarrow$ (1) We must show that 1 has the Egoroff property. Suppose that $c_{n k} \uparrow_{k} 1$. We may assume that $c_{n k} \downarrow_{n}$ (since we can always replace $c_{n k}$ by $c_{1 k} \wedge \ldots \wedge c_{n k}$ ). If there is no sequence $c_{n} \uparrow 1$ such that $c_{n} \neq 1$ for all $n$, we are done; otherwise, let $\left\{c_{n}\right\}$ be such a sequence. Let $a_{n k}=c_{n}^{\prime} \vee c_{n k}^{\prime}$. Clearly $\left.a_{n k}\right\rfloor_{k} c_{n}^{\prime} \vee 1^{\prime}=c_{n}^{\prime}$, and setting $a_{n}=c_{n}^{\prime}$ we have $a_{n k} \downarrow_{k} a_{n} \downarrow 0$. Let $b_{m} \downarrow 0$ be such that $b_{m} \geq a_{n(m), k(m)}$. Certainly $n(m) \rightarrow \infty$, since otherwise we would have some $p$ such that, for infinitely many $m, n(m)=p$ and $b_{m} \geq c_{p}^{\prime} \neq 0$. Finally, let $d_{m}=b_{m}^{\prime}$. Then $d_{m} \uparrow 1$ and, 
for given $m$, $n$, we can find $m^{\prime} \geq m$ such that $n\left(m^{\prime}\right) \geq n$; it follows that $d_{m} \leq d_{m}$ ' $\leq a_{n\left(m^{\prime}\right), k\left(m^{\prime}\right)}^{\prime}=c_{n\left(m^{\prime}\right)} \wedge c_{n\left(m^{\prime}\right), k\left(m^{\prime}\right)} \leq c_{n, k\left(m^{\prime}\right)}$

We denote the symmetric difference of two elements $a$ and $b$ of a Boolean algebra $B$ by $a \Delta b$, i.e. $a \Delta b=\left(a \wedge b^{\prime}\right) \vee\left(a^{\prime} \wedge b\right)$. We shall use below the relation $a \Delta c \leq(a \Delta b) \vee(b \Delta c)$.

Definition 2.3. We say that a sequence $\left\{a_{n}\right\}$ in $B$ is order convergent to an element $a$ of $B$ (notation: $a_{n} \rightarrow a$ ) whenever there exist $b_{n} \downarrow 0$ such that $b_{n} \geq$ $a_{n} \Delta a$. Note that, if $a_{n} \uparrow a$ or $a_{n} \downarrow a$, then $a_{n} \rightarrow a$. Given a subset $A$ of $B$, the derived set $A^{\prime}=\left\{a: a_{n} \rightarrow a\right.$ for some sequence $\left.\left\{a_{n}\right\} \subset A\right\}$ is called the pseudo order closure of $A$. Clearly, $A^{\prime} \supset A$.

Theorem 2.4. If $B$ is a Boolean algebra, then the following statements are equivalent.

(1) $B$ bas the Egoroff property.

(2) For every $a \in B$, if $a_{n k} \rightarrow a_{n} \rightarrow a$, then there is a sequence $\{k(n)\}$ of

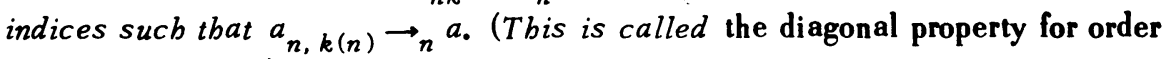
convergence in $B$.)

(3) $\left(A^{\prime}\right)^{\prime}=A^{\prime}$ for every subset $A$ of $B$.

Proof. (1) $\Rightarrow$ (2) Let $a \in B$ and $a_{n k} \rightarrow a_{n} \rightarrow a$. We have $c_{n} \downarrow 0$ such that $a_{n} \Delta a \leq c_{n}$, and $c_{n k} \downarrow_{k} 0$ such that $a_{n k} \Delta a_{n} \leq c_{n k}$. By complementation, the Egoroff property for 1 implies that, for some $b_{n} \downarrow 0, b_{n} \geq c_{n, k(n)}$. Hence $a_{n, k(n)} \Delta a$ $\leq\left(a_{n, k(n)} \Delta a_{n}\right) \vee\left(a_{n} \Delta a\right) \leq b_{n} \vee c_{n} \downarrow 0$.

(2) $\Rightarrow$ (3) Certainly $\left(A^{\prime}\right)^{\prime} \supset A^{\prime}$. On the other hand, if $a \in\left(A^{\prime}\right)^{\prime}$, then for appropriate $a_{n k} \in A, a_{n k} \rightarrow_{k} a_{n} \rightarrow a$. By (2), $a_{n, k(n)} \rightarrow_{n} a$, so that $a \in A^{\prime}$.

(3) $\Rightarrow$ (1) Suppose that $a_{n k} \downarrow_{k} a_{n} \downarrow 0$. Then $0 \in\left(A^{\prime}\right)^{\prime}$, where $A=\left\{a_{n k}: n, k=\right.$ $1,2, \ldots\}$. Hence $0 \in A^{\prime}$, i.e. $a_{n(m), k(m)} \rightarrow_{m} 0$ for certain choices of $n(m), k(m)$. Hence, for some $b_{m} \downarrow 0, b_{m} \geq a_{n(m), k(m)} \Delta 0=a_{n(m), k(m)}$, and (1) follows by Lemma 2.2.

Definition 2.5. It is easy to see that the sets $C \subset B$ such that $C^{\prime}=C$ satisfy the axioms for the closed sets of a topology on $B$. This we call the order topology for the Boolean algebra $B$. The order closure of a set $A \subset B$ is the closure of $A$ with respect to the order topology, and we denote it by $\bar{A}$.

Clearly, $A \subset A^{\prime} \subset\left(A^{\prime}\right)^{\prime} \subset \ldots \subset \bar{A}$, and $A^{\prime}=\bar{A}$ if and only if $A^{\prime}$ is closed, i.e. $\left(A^{\prime}\right)^{\prime}=A^{\prime}$. The following result is therefore a direct consequence of Theorem 2.4.

Theorem 2.6. A Boolean algebra $B$ bas the Egoroff property if and only if the order closure of each subset of B coincides with its pseudo order closure.

3. Egoroff properties and order convergence in Riesz spaces. A Riesz space (a linear vector lattice) is a real linear vector space with a compatible lattice structure. In the following $L$ will always denote a Riesz space and $f, g, \cdots, u$, 
$v, \cdots$ its elements. For every element $f$ in $L$, we write $f^{+}=f \vee 0, f^{-}=(-f) \vee 0$ and $|f|=f \vee(-f)$. The subset $L^{+}=\{f \in L: f \geq 0\}$ is called the positive cone of $L$ and its elements $u, v, \cdots$ positive elements. For the general properties of Riesz spaces, we refer the reader to [10].

Definition 3.1. An element $f$ of a Riesz space $L$ is said to have the Egoroff property if, given any double sequence $\left\{u_{n k}: n, k=1,2, \ldots\right\}$, with $0 \leq u_{n k} \uparrow_{k}|f|$ for each $n$, there exists a sequence $\left\{v_{m}: m=1,2, \ldots\right\}$ such that $0 \leq v_{m} \uparrow|f|$ and for every pair of indices $m, n$, there is an index $k(m, n)$ such that $v_{m} \leq u_{n, k(m, n)^{*}}$ A Riesz space is said to have the Egoroff property if every one of its elements has the Egoroff property. It is equivalent to require that every positive element of the space has the Egoroff property.

There is a close relation between the Egoroff property in Riesz spaces and the Egoroff property in Boolean algebras. Indeed, a Dedekind $\sigma$-complete Riesz space $L$ has the Egoroff property if and only if every principal projection band in the Boolean algebra of all projection bands of $L$ has the Egoroff property (Theorem 74.5 of [10]).

In a Riesz space, there is not in general a replacement for the unit element 1 in a Boolean algebra. Hence, in studying the order topology of a Riesz space, it is natural to introduce the following strong Egoroff property.

Definition 3.2. A Riesz space $L$ is said to have the strong Egoroff property if, whenever $u_{n k} \downarrow_{k} 0$ for all $n$, there exist $v_{m} \downarrow 0$ such that, for every $m, n, v_{m} \geq$ $u_{n, k(m, n)}$ for some index $k(m, n)$.

It follows easily that a Riesz space possessing the strong Egoroff property has also the Egoroff property. The converse is not necessarily true. For an example, the Riesz space of all real bounded sequences with the pointwise ordering has the Egoroff property but not the strong Egoroff property. However, the two properties are equivalent if the Riesz space has the dominating property as defined below.

Definition 3.3. A Riesz space $L$ is said to have the dominating property, if, whenever $\left.u_{n k}\right\rfloor_{k} 0$ for every $n$, there exists an element $w \in L^{+}$such that, for every $n, u_{n, k(n)} \leq w$ for some $k(n)$.

Theorem 3.4. If $L$ is a Riesz space, then $L$ bas the strong Egoroff property if and only if $L$ has both the Egoroff property and the dominating property.

The proof is straightforward and will be omitted.

A Riesz space $L$ is called Archimedean if, for every $u \in L^{+}$, the sequence $\left\{n^{-1} u: n=1,2, \ldots\right\}$ satisfies $n^{-1} u \downarrow 0$.

Theorem 3.5. If $L$ is an Archimedean Riesz space, then $L$ bas the strong Egoroff property if and only if $L$ bas the dominating property. 
Proof. One implication of the theorem holds always. On the other hand, assume $L$ is Archimedean and has the dominating property. Let $u_{n k} \downarrow_{k} 0$ for every $n$. We may assume that $u_{n k} \uparrow_{n}$ as we can always replace $u_{n k}$ by $u_{1 k} \vee \ldots \vee u_{n k}$. Then $\left.n u_{n k}\right\rfloor_{k} 0$ and so by the dominating property there is $w \in L^{+}$such that $n u_{n, k(n)} \leq w$ for some $k(n)$. Let $v_{n}=n^{-1} w$, then $v_{n} \downarrow 0$ since $L$ is Archimedean and $u_{n, k(n)} \leq v_{n}$ for all $n$. Given indices $m$ and $n$, let $l=\max (m, n)$; then $v_{m} \geq$ $v_{l} \geq u_{l, k(l)} \geq u_{n, k(l)}$. Hence $L$ has the strong Egoroff property.

Lemma 3.6. The following statements are equivalent.

(1) $L$ bas the strong Egoroff property.

(2) If $u_{n k} \downarrow u_{n} \downarrow 0$ in $L$, then there exist $v_{m} \downarrow 0$ such that for every $m, v_{m} \geq$ $u_{n(m), k(m)}$ for some index $n(m), k(m)$.

Proof. (1) $\Rightarrow$ (2) Let $u_{n k} \downarrow u_{n} \downarrow 0$. Then $w_{n k}=u_{n k}-u_{n} \downarrow_{k} 0$ for every $n$; so that there exist $w_{m} \downarrow 0$ such that $w_{m} \geq w_{n, k(m, n)}$ for all $m, n$. Now $v_{m}=w_{m}+$ $u_{m}$ satisfies $v_{m} \downarrow 0$ and $v_{m} \geq u_{m, k(m, m)}$.

(2) $\Rightarrow$ (1) Let $u_{n k} \downarrow_{k} 0$ for every $n$. We may assume that $u_{n k} \uparrow_{n}$ as we can always replace $u_{n k}$ by $u_{1 k} \vee \cdots \vee u_{n k}$. If there is no sequence $u_{n} \downarrow 0$ such that $u_{n} \neq 0$ for all $n$, we are done; otherwise, let $\left\{u_{n}\right\}$ be such a sequence. Now $u_{n k}+u_{n} \downarrow_{k} u_{n} \downarrow 0$, so that by (2) there are $v_{m} \downarrow 0$ such that $v_{m} \geq u_{n(m), k(m)}+$ $u_{n(m)}$. Certainly $n(m) \rightarrow \infty$, since otherwise we would have some $p$ such that, for infinitely many $m, n(m)=p$ and $v_{m} \geq u_{p} \neq 0$. For given $m, n$, we can then find

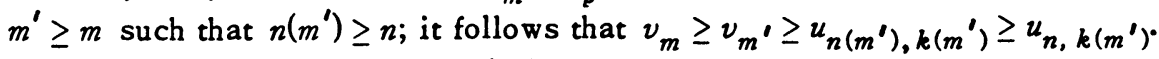

Definition 3.7. A sequence $\left\{f_{n}\right\}$ of elements of a Riesz space $L$ is said to converge in order to an element $f \in L$ if there exist $u_{n} \downarrow 0$ such that $\left|f_{n}-f\right| \leq u_{n}$ holds for all $n$. This will be denoted by $f_{n} \rightarrow_{n} f$. It follows that the limit of an order convergent sequence is unique; moreover, $f_{n} \uparrow f$ or $f_{n} \downarrow f$ implies $f_{n} \rightarrow f$. Given a subset $S$ of $L$, the derived set $S^{\prime}=\left\{f \in L: f_{n} \rightarrow f\right.$ for some sequence $\left.\left\{f_{n}\right\} \subset S\right\}$ is called the pseudo order closure of $S$. It is clear that $S^{\prime} \supset S$.

Definition 3.8. A Riesz space $L$ is said to have the diagonal property for order convergence if, for every $f \in L, f_{n k} \rightarrow_{k} f_{n} \rightarrow f$ implies that $f_{n, k(n)} \rightarrow_{n} f$ for some $k(n)$.

Theorem 3.9. If $L$ is a Riesz space, then the following statements are equivalent.

(1) $L$ bas the strong Egoroff property.

(2) $L$ bas the diagonal property for order convergence.

(3) $\left(S^{\prime}\right)^{\prime}=S^{\prime}$ for every subset $S$ of $L$.

Proof. Similar to the proof of Theorem 2.4. 
Definition 3.10. The subsets $S$ of a Riesz space $L$ such that $S^{\prime}=S$ satisfy the axioms for the closed sets of a topology on $L$. This we call the order topology for $L$. The order closure of a set $S \subset L$ is the closure of $S$ with respect to the order topology, and we denote it by $\bar{S}$.

Clearly, $S \subset S^{\prime} \subset\left(S^{\prime}\right)^{\prime} \subset \ldots \subset \bar{S}$, and $S^{\prime}=\bar{S}$ if and only if $S^{\prime}$ is closed, i.e., $\left(S^{\prime}\right)^{\prime}=S^{\prime}$. Rephrasing part of Theorem 3.9, we have the following.

Theorem 3.11. The following statements are equivalent.

(1) $L$ bas the strong Egoroff property.

(2) The order closure of each subset of $L$ coincides with its pseudo order closure.

We next discuss the interesting fact that the strong Egoroff property is characterized by the behaviour of the order topology on convex subsets of $L$. For a subset $S$ of a Riesz space $L$, we shall denote by $\langle S\rangle$ the convex hull of $S$, i.e., $\langle s\rangle$ is the set of all $f \in L$ of the form $f=\sum_{n=1}^{p} \alpha_{n} f_{n}$ with $\alpha_{1}, \ldots, \alpha_{p}$ real and nonnegative and satisfying $\Sigma_{n=1}^{p} a_{n}=1$, and with $f_{1}, \ldots, f_{p}$ members of $S$.

Lemma 3.12. The following statements are equivalent.

(1) $L$ bas the strong Egoroff property.

(2) If $u_{n k} \downarrow_{k} u_{n} \downarrow 0$ in $L$, then there exist $v_{m} \downarrow 0$ such that, for every $m$, $v_{m} \geq w_{m}$ for some element $w_{m}$ in $\left\langle\left\{u_{n k}\right\}\right\rangle$.

Proof. It is clear that (1) implies (2).

(2) $\Rightarrow$ (1) Let $\left.u_{n k}\right\rfloor_{k} 0$ for every $n$. We may assume that $u_{n k} \uparrow_{n}$ and there exists a sequence $u_{n} \downarrow 0$ such that $u_{n} \neq 0$ for all $n$. We then have $u_{n k} \vee u_{n} \downarrow u_{n} \downarrow 0$; and hence by (2) there exists a sequence $v_{m}^{*} \downarrow 0$ such that, for every $m, v_{m}^{*} \geq w_{m}$ for some element $w_{m}$ in $\left\langle\left\{u_{n k} \vee u_{n}\right\}\right\rangle$.

For a fixed $m, w_{m} \in\left\langle\left\{u_{n k} \vee u_{n}\right\}\right\rangle$ means that we can write $w_{m}=$ $\Sigma_{n, k} a_{n k}^{m}\left(u_{n k} \vee u_{n}\right)$ where $a_{n k}^{m}(n, k=1,2, \ldots)$ are nonnegative real numbers, zero except for finitely many $n$ and $k$, and such that $\Sigma_{n, k} \alpha_{n k}^{m}=1$. For every fixed pair of $m$ and $n$, write $b(m, n)=\max \left\{k: a_{n k}^{m} \neq 0\right\}$ and $a_{n}^{m}=\Sigma_{k} a_{n k}^{m}$; then, for a fixed $m, a_{n}^{m} \geq 0$ for all $n$ with $a_{n}^{m}=0$ except for finitely many $n$ and $\Sigma_{n} a_{n}^{m}=1$. Furthermore, we have

$$
v_{m}^{*} \geq w_{m} \geq \sum_{n} \alpha_{n}^{m}\left(u_{n, b(m, n)} V u_{n}\right) .
$$

We will show that the sequence $\left\{v_{m}=2 v_{m}^{*}: m=1,2, \ldots\right\}$ satisfies $v_{m} \downarrow 0$ and for given pair $M$ and $N$ of indices, there exists an index $k(M, N)$ such that $v_{M} \geq u_{N, k(M, N)}$.

Obviously, $v_{m} \downarrow 0$. Given $M$ and $N$. Let $\gamma=\sup \left\{\Sigma_{n \geq N} a_{n}^{m}: m \geq M\right\}$. We prove that $\gamma=1$. It is clear that $0 \leq \gamma \leq 1$. Moreover, for every $m \geq M$, we have 


$$
\begin{aligned}
v_{m}^{*} & \geq \sum_{n} a_{n}^{m}\left(u_{n, b(m, n)} V u_{n}\right) \geq \sum_{n<N} a_{n}^{m} u_{n} \\
& \geq\left(\sum_{n<N} a_{n}^{m}\right) u_{N}=\left(\sum_{n} \alpha_{n}^{m}-\sum_{n \geq N} a_{n}^{m}\right) u_{N} \geq(1-\gamma) u_{N}
\end{aligned}
$$

but $\inf \left\{v_{m}^{*}: m \geq M\right\}=0$ and $u_{N} \neq 0$ as we have chosen it so, therefore $\gamma=1$. Hence, there exists a positive integer $p \geq M$ such that $\Sigma_{n \geq N} a_{n}^{p} \geq 1 / 2$, and so

$$
2 v_{M}^{*} \geq 2 v_{p}^{*} \geq 2 \sum_{n} a_{n}^{p}\left(u_{n, b(p, n)} V u_{n}\right) \geq 2 \sum_{n \geq N} a_{n}^{p} u_{n, b(p, n)}
$$

if we let $k(M, N)=\max \left\{b(p, n): \alpha_{n}^{p} \neq 0\right\}$ and recall that $u_{n k} \uparrow_{n}$, we thus obtain

$$
2 v_{M}^{*} \geq 2\left(\sum_{n \geq N} a_{n}^{p}\right) u_{N, k(M, N)} \geq u_{N, k(M, N)},
$$

i.e. $v_{M} \geq u_{N, k(M, N)}$ as required.

Corollary 3.13. The following statements are equivalent.

(1) $L$ bas the strong Egoroff property.

(2) For every element $f \in L, f_{n k} \rightarrow f_{n} \rightarrow f$ implies the existence of a sequence $\left\{g_{m}\right\}$ in $\left\langle\left\{f_{n k}\right\}\right\rangle$ such that $g_{m} \rightarrow f$.

An immediate result from the above results is

Theorem 3.14. The following statements are equivalent.

(1) $L$ bas the strong Egoroff property.

(2) The order closure of each convex subset of L coincides with its pseudo order closure.

A subset $S$ of a Riesz space $L$ is called order bounded if there exists an element $u \in L^{+}$such that $|f| \leq u$ for all $f \in S$. In a way, the Egoroff property is the strong Egoroff property restricted to order bounded subsets. This can be clarified by the following theorem. The proof of it is similar to that of the corresponding results relating to the strong Egoroff property.

Theorem 3.15. The following statements are equivalent.

(1) $L$ bas the Egoroff property.

(2) If $u \geq u_{n k} \downarrow_{k} u_{n} \downarrow 0$ in $L$, then there exist $v_{m} \downarrow 0$ such that for every $m$, $v_{m} \geq u_{n(m), k(m)}$ for some indices $n(m)$ and $k(m)$.

(3) If $f_{n k} \rightarrow_{k} f_{n} \rightarrow f$ in $L$ and the set $\left\{f_{n k}\right\}$ is order bounded, then $f_{n(m), k(m)} \rightarrow_{m} f$ for some indices $n(m)$ and $k(m)$.

(4) The order closure of each order bounded subset of $L$ coincides with its pseudo order closure.

(5) The order closure of eacb order bounded convex subset of $L$ coincides with its pseudo order closure. 
The Egoroff property is similar to the notion of total continuity introduced by Nakano [13]. Due to its relation to the classical Egoroff theorem (see Theorems 3.1 and 3.2 of [4]), we take the present definition and terminology as in Luxemburg and Zaanen [7, Definition 20.5, Note VI]. In [5], Kantorovitch introduced the notion of regularity in Riesz spaces, which implies the strong Egoroff property. The diagonal property for order convergence can also be found in [5], [10], [14], [15], [16], [18]. It seems that Nakano was the first to isolate the dominating property, which he called regular completeness ([12] and [13]).

4. Relative uniform convergence in Riesz spaces.

Definition 4.1. A sequence $\left\{f_{n}\right\}$ of elements of a Riesz space $L$ is said to converge r.u. (relatively uniformly) to an element $f \in L$ if there exists an eiement $v \in L^{+}$and a real sequence $a_{n} \downarrow 0$ such that $\left|f_{n}-f\right| \leq a_{n} v$ for all $n$. This will be denoted by $f_{n} \stackrel{r u}{\rightarrow} f$.

The limit of a r.u. convergent sequence is not necessarily unique. It can be proved that the limit of any r.u. convergent sequence in a Riesz space $L$ is unique if and only if $L$ is Archimedean.

Definition 4.2. For any subset $S$ of a Riesz space $L$, the set $S_{r u}^{\prime}=$ $\left\{f \in L: f_{n} \stackrel{r u}{\longrightarrow} f\right.$ for some sequence $\left.\left\{f_{n}\right\} \subset S\right\}$ is called the pseudo r.u. closure of $S$.

Definition 4.3. The subsets $S$ of a Riesz space $L$ such that $S_{r u}^{\prime}=S$ satisfy the axioms for the closed sets of a topology on L. This we call the r.u. topology for $L$. The r.u. closure of a set $S \subset L$ is the closure of $S$ with respect to the r.u. topology and will be denoted by $\bar{S}^{r u}$.

Definition 4.4. A Riesz space $L$ is said to have the $\sigma$-property if, for any sequence $\left\{u_{n}\right\}$ in $L^{+}$, there exists an element $u \in L^{+}$and a sequence $\left\{a_{n}\right\}$ of real numbers such that $u_{n} \leq a_{n} u$ for all $n$.

Lemma 4.5. If $L$ is an Archimedean Riesz space, then the following statements are equivalent.

(1) $L$ bas the $\sigma$-property. $k(n)$.

(2) For every $f \in L, f_{n k} \stackrel{r u}{\longrightarrow} f_{n} \stackrel{r u}{\longrightarrow} f$ implies that $f_{n, k(n)} \stackrel{r u}{\longrightarrow}$ f for some

(3) For every $f \in L, f_{n k} \stackrel{r u}{\longrightarrow} f_{n} \stackrel{r u}{\longrightarrow} f$ implies the existence of a sequence $\left\{g_{m}\right\}$ in $\left\langle\left\{f_{n k}\right\}\right\rangle$ sucb tbat $g_{m} \stackrel{r u}{\longrightarrow} f$.

Proof. (1) $\Rightarrow$ (2) Let $f_{n k} \stackrel{r u}{\longrightarrow} f_{n} \stackrel{r u}{\longrightarrow} f$. Then $\left|f_{n k}-f_{n}\right| \leq \alpha_{n k} u_{n}$ and $\left|f_{n}-f\right| \leq a_{n} u$ for some sequence $\left\{u_{n}\right\} \subset L^{+}$and some element $u \in L^{+}$, and for some real number sequences $\alpha_{n k} \downarrow_{k} 0$ and $\alpha_{n} \downarrow 0$. By (1), there is an element $w \in L^{+}$and a sequence $\left\{\beta_{n}\right\}$ of real numbers such that $u_{n} \leq \beta_{n} w$ for all $n_{\text {. Since }}$ $\alpha_{n k} \beta_{n} \downarrow_{k} 0$ for every $n$, by the strong Egoroff property of the real numbers, there 
is a sequence $\gamma_{n} \downarrow 0$ such that $a_{n, k(n)} \beta_{n} \leq \gamma_{n}$ for some $k(n)$. Now we have

$$
\begin{aligned}
\left|f_{n, k(n)}-f\right| & \leq\left|f_{n, k(n)}-f_{n}\right|+\left|f_{n}-f\right| \leq a_{n, k(n)} u_{n}+a_{n} u \\
& \leq a_{n, k(n)} \beta_{n} w+a_{n} u \leq \gamma_{n} w+\alpha_{n} u \leq\left(\gamma_{n}+\alpha_{n}\right)(w V u)
\end{aligned}
$$

and $\dot{\gamma}_{n}+a_{n} \downarrow 0$, hence $f_{n, k(n)} \stackrel{r u}{\longrightarrow} f$.

It is clear that $(2) \Rightarrow(3)$.

(3) $\Rightarrow$ (1) Let $\left\{u_{n}\right\}$ be an arbitrary sequence in $L^{+}$. We shall show that there is an element $w \in L^{+}$and a real sequence $\left\{\gamma_{n}\right\}$ such that $u_{n} \leq \gamma_{n} w$ for all $n_{\text {. }}$ There is no loss in generality to assume that $u_{n} \uparrow_{n}$.

Let $u$ be a nonzero element of $L^{+}$. We set

$$
\begin{aligned}
& w_{n k}=k^{-1} u_{n}+n^{-1} u \quad \text { for } n, k=1,2, \ldots, \\
& w_{n}=n^{-1} u
\end{aligned}-\frac{\text { for } n}{2}=1,2, \ldots .
$$

Then $w_{n k} \stackrel{r u}{\longrightarrow} w_{n} \stackrel{r u}{\longrightarrow} 0$. Hence, by (3), there is a sequence $\left\{v_{m}\right\}$ in $\left\langle\left\{w_{n k}\right\}\right\rangle$ such that $v_{m} \stackrel{r u}{\longrightarrow} 0$.

For each fixed $m$, since $v_{m}$ is a member of $\left\langle\left\{w_{n k}\right\}\right\rangle$, it is a finite linear combination of elements from $\left\{w_{n k}: n, k=1,2, \ldots\right\}$ with nonnegative coefficients the sum of which equals one. Hence, denoting by $a_{n}^{m}$ the sum of the coefficients belonging to the same $n$, we have $a_{n}^{m} \geq 0$ for all $n, a_{n}^{m}=0$ except for finitely many $n, \Sigma_{n} a_{n}^{m}=1$ and

$$
v_{m} \geq \sum_{n} \alpha_{n}^{m} w_{n, k(m, n)}
$$

for an appropriate $k(m, n)$. It follows from $v_{m} \stackrel{r u}{\longrightarrow} 0$ that there is an element $w \in L^{+}$and a real sequence $\beta_{m} \downarrow 0$ such that $v_{m} \leq \beta_{m} w$ for all $m$. Then $\beta_{m} w \geq$ $\Sigma_{n} a_{n}^{m} w_{n, k(m, n)}$ holds for all $m_{0}$

Denote by $Q$ the set of all natural numbers $n$ such that $a_{n}^{m} \neq 0$ for some $m$. It is clear that $\mathbb{Q}$ is nonempty. We prove that $\mathcal{Q}$ has infinitely many elements. Suppose $\mathcal{Q}$ is finite and let $p$ be the largest number in $\mathcal{Q}$. Then, for every $m$, we have

$$
\beta_{m} w \geq \sum_{n} a_{n}^{m} w_{n, k(m, n)} \geq \sum_{n} a_{n}^{m} n^{-1} u \geq \sum_{n} a_{n}^{m} p^{-1} u=p^{-1} u ;
$$

on the other hand $\beta_{m} w \downarrow 0$ since $L$ is Archimedean, and so $p^{-1} u \leq 0$ which contradicts that $u>0$.

It remains to show that for a given natural number $N$, there is some real number $\gamma_{N}$ such that $u_{N} \leq \gamma_{N} w$. Let $N$ be given. By the above argument that $\mathcal{Q}$ is an infinite set, there is a natural number $n_{0}=n_{0}(N)$ in $\mathbb{Q}$ such that $n_{0} \geq N$; so $a_{n_{0}}^{m_{0}} \neq 0$ for some index $m_{0}=m_{0}(N)$. We then have 


$$
\begin{aligned}
\beta_{m_{0}} w & \geq \sum_{n} \alpha_{n}^{m 0} w_{n, k\left(m_{0}, n\right)} \geq \sum_{n} \alpha_{n}^{m_{0}} \cdot\left(k\left(m_{0}, n\right)\right)^{-1} u_{n} \\
& \geq \alpha_{n_{0}}^{m_{0}} \cdot\left(k\left(m_{0}, n_{0}\right)\right)^{-1} u_{n_{0}} .
\end{aligned}
$$

Set $\gamma_{N}=\beta_{m_{0}} \cdot\left(\alpha_{n_{0}}^{m_{0}}\right)^{-1} \cdot k\left(m_{0}, n_{0}\right)$ and recall that $u_{n} \uparrow_{n}$, we thus have $\gamma_{N} w \geq$ $u_{N}$. This completes the proof.

A Riesz space $L$ is said to have the diagonal propetry for r.u. convergence if $L$ satisfies condition (2) of Lemma 4.5. By this lemma, we have

Theorem 4.6. If $L$ is an Archimedean Riesz space, then the following state. ments are equivalent.

(1) $L$ bas the $\sigma$-property.

(2) $L$ bas the diagonal property for r.u. convergence.

(3) The r.u. closure of each subset of L coincides with its pseudo r.u. closure.

(4) The r.u. closure of each convex subset of $L$ coincides with its pseudo r.u. closure.

In conclusion, we point out several facts whose proofs can be found in [10] concerning the strong Egoroff property and the $\sigma$-property. An Archimedean Riesz space which has the strong Egoroff property also has the $\sigma$-property. The converse does not always hold (see Exercise 16.18 of [10]). We say that a mode of convergence of sequences is stable if for an arbitrary sequence $\left\{f_{n}\right\}$ which converges to 0 there exists a real sequence $a_{n} \uparrow \infty$ such that the sequence $\left\{a_{n} f_{n}\right\}$ also converges to 0 . R.u. convergence in a Riesz space is stable. Order convergence in a Riesz space is not necessarily stable. If a Riesz space $L$ has the strong Egoroff property, then order convergence in $L$ is stable. For an Archimedean Riesz space $L, L$ has the strong Egoroff property if and only if $L$ has the $\sigma$-property and order convergence in $L$ is stable.

\section{REFERENCES}

1. G. Birkhoff, On the structure of abstract algebras, Proc. Cambridge Philos. Soc. 31 (1935), 433-454.

2. —, Lattice theory, 3rd ed., Amer. Math. Soc. Colloq. Publ., vol. 24, Amer. Math. Soc., Providence, R. I., 1967. MR 37 \#2638.

3. D. T. Egoroff, Sur les suites des fonctions measurables, C. R. Acad. Sci. Paris 152 (1911), 244-246.

4. J. A. R. Holbrook, Seminorms and the Egoroff property in Riesz spaces, Trans. Amer. Math. Soc. 132 (1968), 67-77. MR 37 \# 4558.

5. L. Kantorovitch, Lineare halbgeordnete Räume, Trans. Moscow Math. Soc. 44 (1937), 121-165. 
6. W. A. J. Luxemburg and L. C. Moore, Jr., Archimedean quotient Riesz spaces, Duke Math. J. 34 (1967), 725-739. MR 36 \#651.

7. W. A. J. Luxemburg and A. C. Zaanen, Notes on Banach function spaces, I-XIII, Nederl. Akad. Wetensch. Proc. Ser. A 66 = Indag. Math. 25 (1963), 135-147, 148-153, 239-250, 251-263, 496-504, 655-668, 669-681; ibid. Ser. A 67 = Indag. Math. 26 (1964), 104-119, 360-376, 493-506, 507-518, 519-529, 530-543. MR 26 \#6723 a, b; 27 \#5119a,b; 28 \# 1481; 28 \#5324 a, b; 30 \#3381 a, b.

8. W. A. J. Luxemburg, Notes on Banach function spaces, XIVa, b, XVa, b, XVIa, b, Nederl. Akad. Wetensch. Proc. Ser. A 68 = Indag. Math. 27 (1965), 229-248, 415-446, 646-667. MR 32 \#6202a, b, c, d, e.

9. - On finitely additive measures in Boolean algebras, J. Reine Angew. Math. 213 (1963/64), 165-173. MR 29 \#1158.

10. W. A. J. Luxemburg and A. C. Zaanen, Riesz spaces. Vol. I, North-Holland, Amsterdam-London, 1971.

11. E. H. Moore, On the foundations of a theory of linear integral equations, Bull. Amer. Math. Soc. 18 (1912), 334-362.

12. H. Nakano, Über ein lineares Funktional auf dem teilweise geordneten Modul, Proc. Imp. Acad. Tokyo, 18 (1942), 548-552. MR 7, 249.

13. —, Linear lattices, 2nd ed., Wayne State Univ. Press, Detroit, Mich., 1966. MR 33 \# 3084.

14. M. Orihara, On the regular vector lattice, Proc. Acad. Tokyo 18 (1942), 525-529. MR 7, 250.

15. A. L. Peressini, Ordered topological vector spaces, Harper and Row, New York, 1967. MR $37 \# 3315$.

16. A. L. Peressini and D. R. Sherbert, Order properties of linear mappings on sequence spaces, Math, Ann. 165 (1966), 318-332. MR 33 \#6363.

17. B. Z. Vulih, Introduction to the theory of partially ordered spaces, Fizmatgiz, Moscow, 1961; English transl., Noordhoff, Groningen, 1967. MR 24 \# A3494; 37 \# 121.

18. A. C. Zaanen, Stability of order convergence and regularity in Riesz spaces, Studia Math. 31 (1968), 159-172. MR 39 \#1944.

DEPARTMENT OF MATHEMATICS, UNIVERSITY OF ALBERTA, EDMONTON, ALBERTA, CANADA

DEPARTMENT OF MATHEMATICS, FLINDERS UNIVERSITY OF SOUTH AUSTRALIA, BEDFORD PARK 5042, SOUTH AUSTRALIA, AUSTRALIA (Current address) 